\title{
Tightness Criterion and Weak Convergence for the Generalized Empirical Process in $D[0,1]$
}

\author{
Maciej Ziemba \\ Department of Mathematics, Lublin University of Technology, ul. Nadbystrzycka 38d, \\ 20-618 Lublin, Poland \\ Correspondence should be addressed to Maciej Ziemba; maciek.ziemba@gmail.com
}

Received 27 June 2013; Accepted 23 August 2013

Academic Editors: M. Campanino, S. Lototsky, H. J. Paarsch, and L. Sacerdote

Copyright (C) 2013 Maciej Ziemba. This is an open access article distributed under the Creative Commons Attribution License, which permits unrestricted use, distribution, and reproduction in any medium, provided the original work is properly cited.

We prove Shao and Yu's tightness criterion for the generalized empirical process in the space $D[0,1]$ with $J_{1}$ topology. Covariance inequalities are used in applying the criterion to particular types of the empirical processes. We weaken the assumptions imposed on the covariance structure as well as the properties of the underlying sequence of r.v.s, under which presented processes converge weakly.

\section{Introduction}

Let $\left\{X_{n}\right\}_{n \geq 1}$ be a sequence of absolutely continuous identically distributed (i.d.) random variables (r.v.s) with an unknown distribution function (d.f.) $F$ and probability density function (p.d.f.) $f$. The empirical distribution function, based on the first $n$ r.v.'s, is defined by $F_{n}(x)=n^{-1} \sum_{j=1}^{n} I\left[X_{j} \leq x\right]$. It is well known, however, that this estimate does not make use of the smoothness of $F$, that is, the existence of the p.d.f. $f$. Therefore, the kernel estimate

$$
\bar{F}_{n}(x)=\frac{1}{n} \sum_{j=1}^{n} K\left(\frac{x-X_{j}}{h_{n}}\right)
$$

has been proposed, where the kernel function $K$ is a known d.f. and $\left\{h_{n}\right\}_{n \geq 1}$ is a sequence of positive constants descending at an appropriate rate. Such estimator has been deeply studied in the last two decades mainly by Cai and Roussas in [1-4], $\mathrm{Li}$ and Yang in [5] and others. Asymptotic normality, BerryEssen bounds for smooth estimator $\bar{F}_{n}(x)$ are only examples of their fruitful results.

Recently, Li et al. proposed in [6] the so-called recursive kernel estimator of the d.f. $F$ as follows:

$$
\widehat{F}_{n}(x)=\frac{1}{n} \sum_{j=1}^{n} K\left(\frac{x-X_{j}}{h_{j}}\right) .
$$

The seemingly tiny modification they introduced to the formula of the typical kernel estimator has an important advantage. Namely, in the case of a large size of a sample, $\widehat{F}_{n}(x)$ can be easily updated with each new observation since it is computable recursively by

$$
\widehat{F}_{n}(x)=\frac{n-1}{n} \widehat{F}_{n-1}(x)+\frac{1}{n} K\left(\frac{x-X_{n}}{h_{n}}\right),
$$

where $\widehat{F}_{0}(x)=0$. The authors discussed the asymptotic bias and quadratic-mean convergence and established the pointwise asymptotic normality of $\widehat{F}_{n}(x)$ under relevant assumptions.

In this paper, however, we will focus on the empirical process built on an estimator $F_{n}(x)$ of the d.f. $F$ rather than $F_{n}(x)$ itself. Let us recall that the following process:

$$
\alpha_{n}(x)=\sqrt{n}\left[F_{n}(x)-E F_{n}(x)\right], \quad \text { where } x \in \mathbf{R}
$$

is called the empirical process built on an estimator $F_{n}(x)$.

$\mathrm{Yu}$ [7] studied the case when $F_{n}(x)$ is a standard empirical d.f. and showed weak convergence of $\alpha_{n}(\cdot)$ to the Gaussian process assuming stationarity and association of the underlying r.v.s. Cai and Roussas [1] obtained a similar result in the case when $F_{n}(x)$ is the kernel estimator of the d.f. $F$ built on a stationary sequence of negatively associated r.v.s. 
In this paper, we shall study the empirical process $\alpha_{n}(x)$ generated by the generalized kernel estimator of the d.f. given by the formula

$$
\widetilde{F}_{n}(x)=\frac{1}{n} \sum_{j=1}^{n} K\left(\frac{x-X_{j}}{h_{n, j}}\right), \quad \text { where }
$$

A1: $\left\{X_{j}\right\}_{j \geq 1}$ is a sequence of absolutely continuous i.d. r.v.s taking values in $[0,1]$ and having twice differentiable d.f. $F$ with first and second derivative bounded;

A2: $K$ is a kernel function such that $\int_{\mathbf{R}} u d K(u)=0$ and $\int_{\mathbf{R}} u^{2} d K(u)<\infty$ with bounded derivative $k$;

A3: $\left\{h_{n, j}\right\}_{n \geq 1, j \in\{1, \ldots, n\}}$ is a sequence of positive constants subject to the following conditions: $\lim _{j, n \rightarrow \infty} h_{n, j}=0$, $\lim _{j, n \rightarrow \infty} n h_{n, j}^{4}=0$ (actually, since $j \leq n, j \rightarrow \infty$ under the limit suffices).

Explicitly, we take a look onto the process

$$
\alpha_{n}(x)=\frac{1}{\sqrt{n}} \cdot \sum_{j=1}^{n}\left[K\left(\frac{x-X_{j}}{h_{n, j}}\right)-E K\left(\frac{x-X_{j}}{h_{n, j}}\right)\right],
$$

we shall call from now on the generalized empirical process. Let us pay attention to the fact that in the case of

(i) $h_{n, j}=h_{n}$ for $j \in\{1, \ldots, n\}, \alpha_{n}(x)$ is the empirical process based on the kernel estimator of the d.f. $F$;

(ii) $h_{j, j}=h_{j+1, j}=h_{j+2, j}=\cdots=: h_{j}$ for $j \in \mathbf{N}, \alpha_{n}(x)$ is the empirical process based on the recursive kernel estimator of the d.f. F;

(iii) $K(t)=I_{[0, \infty]}(t), \alpha_{n}(x)$ is the standard empirical process (based on the empirical d.f.).

It is well known that the crucial procedure in showing weak convergence for an empirical process is to verify tightness. In [8], Shao and Yu gave the following criterion:

$$
\begin{gathered}
\exists_{C_{1}>0} \exists_{p>2} \exists_{p_{1}>1} \exists_{0 \leq r_{1} \leq 1} \exists_{p_{2}>1-r_{1}} \forall_{x, y \in[0,1]} \\
E\left|\alpha_{n}(x)-\alpha_{n}(y)\right|^{p} \leq C_{1}\left(|x-y|^{p_{1}}+n^{-p_{2} / 2}|x-y|^{r_{1}}\right),
\end{gathered}
$$

under which the standard empirical process based on stationary sequence of uniform $[0,1]$ r.v.s is tight. It is stated there that the proof of that fact is an easy standard procedure parallel to the one presented in [9]. It is the main aim of this paper to carry it in details but for the generalized empirical process defined by (6) and without assuming stationarity. Nevertheless, we will always return to stationarity assumption while establishing weak convergence.

In order to obtain tightness, one has to assume appropriate covariance structure of the underlying r.v.s, that is, the covariance of a pair of r.v's $X_{i}$ and $X_{j}$ has to decline at the right rate while $i$ and $j$ are growing apart. In this paper we lower the demanded rate of covariance decay using the covariance inequalities for associated (c.f. $[10,11])$ and multivariate totally positive of order $2\left(\mathrm{MTP}_{2}\right)$ (c.f. [12]) r.v.s obtained in [13].
The paper is organized as follows. In Section 2 we present the proof of the Shao and Yu's tightness criterion formulated for our generalized empirical process. Sections 3 and 4 are devoted to application of the criterion to showing tightness and thus weak convergence of the specific types of empirical processes. Section 5 concerns weak convergence of the recursive kernel-type process for i.i.d. r.v.s.

\section{Tightness Criterion}

We start with the key point of the paper.

Theorem 1. Let $\left\{\alpha_{n}(x)\right\}_{n \geq 1} \in D[0,1]$ be the generalized empir$i$ al process defined as in (6). One assume that A1, A2, A3 hold.

If there exist constants $C>0, p>2, p_{1}>1,0 \leq p_{3} \leq 1$, $p_{2}>1-p_{3}$, such that for any $x, y \in[0,1]$ and $n \in \mathbf{N}$ the following inequality holds:

$$
E\left|\alpha_{n}(x)-\alpha_{n}(y)\right|^{p} \leq C \cdot\left(|x-y|^{p_{1}}+n^{-p_{2} / 2} \cdot|x-y|^{p_{3}}\right),
$$

then the process $\left\{\alpha_{n}(x)\right\}_{n \geq 1}$ is tight in $D[0,1]$ with $J_{1}$ topology.

Proof. The proof boils down to showing that under the assumptions made in Theorem 1, conditions of Theorem 13.2 in [9] hold. Let us recall that in light of the above mentioned theorem, a process $\left\{\alpha_{n}(x)\right\}_{n \geq 1}$ is tight in $D[0,1]$ if

$$
\begin{gathered}
\lim _{a \rightarrow \infty} \limsup _{n \rightarrow \infty} P\left(\left\|\alpha_{n}\right\| \geq a\right)=0, \\
\lim _{\delta \rightarrow 0} \limsup _{n \rightarrow \infty} P\left(w_{1}^{\prime}\left(\alpha_{n}, \delta\right) \geq \epsilon\right)=0 \quad \forall \epsilon>0,
\end{gathered}
$$

where $\|\cdot\|$ is the supremum norm, that is,

$$
\left\|\alpha_{n}\right\|=\sup _{0 \leq x \leq 1}\left|\alpha_{n}(x)\right|
$$

and $w_{1}^{\prime}\left(\alpha_{n}, \delta\right)$ is the modulus of continuity of the function $\alpha_{n} \in D[0,1]$, that is

$$
w_{1}^{\prime}\left(\alpha_{n}, \delta\right):=\inf _{\left\{t_{i}\right\}} \max _{1 \leq i \leq v} \sup _{x, y \in\left[t_{i-1}, t_{i}\right)}\left|\alpha_{n}(x)-\alpha_{n}(y)\right| .
$$

The infimum runs over all finite " $\delta$-sparse" decompositions $\left\{\left[t_{i-1}, t_{i}\right), i \in 1, \ldots, v\right\}$ of the interval $[0,1]$. In other words, it runs over all choices of increasingly ordered points $\left\{t_{i}\right\}_{1 \leq i \leq v}$ such that $\min _{1 \leq i \leq \nu}\left(t_{i}-t_{i-1}\right)>\delta$, where $t_{0}=0, t_{v}=1$.

Let us first show that condition (9) is satisfied. According to the corollary following Theorem 13.2 in [9], it suffices to show

$$
\lim _{a \rightarrow \infty} \limsup _{n \rightarrow \infty} P\left(\alpha_{n}:\left|\alpha_{n}(x)\right| \geq a\right)=0 \quad \forall x \in[0,1],
$$

that is,

$$
\forall_{\eta \geq 0} \exists_{a>0} \forall_{n \in \mathbf{N}} P\left(\alpha_{n}:\left|\alpha_{n}(x)\right| \geq a\right) \leq \eta \quad \forall x \in[0,1] .
$$

Let us fix $x \in[0,1]$ and $\eta>0$. We need to find $a=a(\eta)>0$, such that

$$
P\left(\sqrt{n}\left|\widetilde{F}_{n}(x)-E \widetilde{F}_{n}(x)\right| \geq a\right) \leq \eta \quad \forall n \in \mathbf{N},
$$


where

$$
\widetilde{F}_{n}(x)=\frac{1}{n} \sum_{j=1}^{n} K\left(\frac{x-X_{j}}{h_{n, j}}\right)
$$

is the generalized kernel estimator of the d.f. F. Applying Chebyshev's inequality we shall find $a$ satisfying

$$
a \geq \frac{\sqrt{n}}{\eta} \cdot E\left|\widetilde{F}_{n}(x)-E \widetilde{F}_{n}(x)\right| \quad \forall n \in \mathbf{N} .
$$

Such $a$ exists if $E\left|\alpha_{n}(x)\right|<\infty$ for all $n \geq 1$. Implementing $y=0$ and $p_{3}=0$ in the assumption (8), we get for $p>2$, $p_{1}, p_{2}>1$

$$
E\left|\alpha_{n}(x)\right|^{p} \leq C \cdot\left(|x|^{p_{1}}+n^{-p_{2} / 2}|x|^{p_{3}}\right) \leq C \cdot\left(1+\frac{1}{n^{p_{2} / 2}}\right) \leq 2 C .
$$

Now, applying Hölder's inequality, we arrive at

$$
E\left|\alpha_{n}(x)\right| \leq \sqrt[4]{E\left|\alpha_{n}(x)\right|^{4}}
$$

which in light of the inequality (18) for $p=4$, is bounded from above by $\sqrt[4]{2 C}<\infty$. Therefore condition (9) holds.

We shall now proceed to checking condition (10). Let us recall that the modulus of continuity of the function $x \in$ $C[0,1], \delta>0$, is given by the formula

$$
w_{1}(x, \delta)=\sup _{0 \leq y \leq 1-\delta} \sup _{y \leq x \leq y+\delta}\left|\alpha_{n}(x)-\alpha_{n}(y)\right| .
$$

As it is shown in [9] (page 123), there is a relation between $w_{1}(\cdot, \cdot)$ and $w_{1}^{\prime}(\cdot, \cdot)$ in the spaces $C[0,1]$ and $D[0,1]$ relatively. Namely, for $x \in D[0,1]$ and $\delta<1 / 2$

$$
w_{1}(x, 2 \delta) \geq w_{1}^{\prime}(x, \delta) \text {. }
$$

Thus,

$$
P\left(w_{1}^{\prime}\left(\alpha_{n}, \delta\right) \geq \epsilon\right) \leq P\left(w_{1}\left(\alpha_{n}, 2 \delta\right) \geq \epsilon\right) .
$$

Therefore, condition (10) holds if we show

$$
\forall_{\epsilon>0} \forall_{\eta>0} \exists_{0 \leq \delta<1 / 2} \exists_{n_{0}} \forall_{n \geq n_{0}} P\left(w_{1}\left(\alpha_{n}, 2 \delta\right) \geq \epsilon\right) \leq \eta .
$$

With a view to obtaining the desired inequality we shall proceed patiently in five steps.

Step 1 . We need a moment inequality for the r.v. $\alpha_{n}(x)-\alpha_{n}(y)$ involving the distance between the points $x$ and $y$. Let us then assume that for the constants $C, p, p_{1}, p_{2}, p_{3}$ given in Theorem 1, inequality (8) holds. Fix $\epsilon>0, \eta>0$ and define the quantity $r_{n}=\epsilon / \sqrt{n}, n \in \mathbf{N}$. Next, we fix $x, y \in[0,1]$ and take $n$ large enough so that $r_{n} \leq|x-y|$. Then $n^{-1 / 2} \leq|x-y| \epsilon^{-1}$ and we have

$$
E\left|\alpha_{n}(x)-\alpha_{n}(y)\right|^{p} \leq C\left(1+\epsilon^{-p_{2}}\right)|x-y|^{\min \left\{p_{1}, p_{2}+p_{3}\right\}} .
$$

Step 2. Let us now fix $n \in \mathbf{N}, \delta \in(0,1)$ and consider the following r.v.s:

$$
\chi_{i}=\alpha_{n}\left(y+i r_{n}\right)-\alpha_{n}\left(y+(i-1) r_{n}\right)
$$

for $i \in 1,2, \ldots, m_{n}$, where $m_{n}=m(n, \delta)$ is such that $r_{n} m_{n} \leq \delta$. It is easy to see that for $S_{i}:=\sum_{k=1}^{i} \chi_{k}$

$$
\max _{1 \leq i \leq m_{n}}\left|S_{i}\right|=\max _{1 \leq i \leq m_{n}}\left|\alpha_{n}\left(y+i r_{n}\right)-\alpha_{n}(y)\right|
$$

Let us notice that for r.v's $\left\{\chi_{i}\right\}_{i \geq 1}$ the conditions of Theorem 10.2 in [9] are satisfied with $4 \beta=p, u_{l}=r_{n} \forall_{i<l \leq j}$ and $2 \alpha=\min \left\{p_{1}, p_{2}+p_{3}\right\}$. Therefore, we are equipped with the following maximal inequality:

$$
\begin{gathered}
P\left(\max _{1 \leq i \leq m_{n}}\left|\alpha_{n}\left(y+i r_{n}\right)-\alpha_{n}(y)\right| \geq \lambda\right) \\
\leq \frac{C_{p, \min \left\{p_{1}, p_{2}+p_{3}\right\}}}{\lambda^{p}}\left(m_{n} r_{n}\right)^{\min \left\{p_{1}, p_{2}+p_{3}\right\}}
\end{gathered}
$$

for all $\lambda>0$.

Step 3. Let $M=\sup _{x \in[0,1]} f(x)$, where $f$ is the p.d.f. of r.v's $\left\{X_{j}\right\}_{j \geq 1}$. For fixed $\epsilon, \eta>0$ let us take $\delta>0$ such that

$$
\frac{C_{p, \min \left\{p_{1}, p_{2}+p_{3}\right\}}}{(M \epsilon)^{p}} \cdot(2 \delta)^{\min \left\{p_{1}, p_{2}+p_{3}\right\}}<\eta
$$

and define $m_{n}:=\left\lfloor\delta / r_{n}\right\rfloor$. For sufficiently large $n \in \mathbf{N}$ we have $m_{n} \geq 1$ and then we get

$$
r_{n} m_{n} \leq \delta<\left(m_{n}+1\right) r_{n} \leq 2 m_{n} r_{n} \leq \delta
$$

Step 4. Our goal is to obtain an inequality which enables us to bound the supremum of the increment of the function $\alpha_{n}$ via the maximum of the increments of that function on some subintervals. To be more precise, we will find the upper bound for

$$
\sup _{y \leq x \leq y+m_{n} q}\left|\alpha_{n}(x)-\alpha_{n}(y)\right|
$$

in terms of

$$
\max _{1 \leq i \leq m_{n}}\left|\alpha_{n}(y+i q)-\alpha_{n}(y)\right|
$$

where $x, y \in[0,1], y \leq x \leq y+q, q \geq \epsilon / n$ and $m_{n}$ is defined as in Step 3.

Let us recall that $\alpha_{n}(x)=\sqrt{n}\left(\widetilde{F}_{n}(x)-E \widetilde{F}_{n}(x)\right)$, where $\widetilde{F}_{n}(x)=(1 / n) \sum_{j=1}^{n} K\left(\left(x-X_{j}\right) / h_{n, j}\right)$. From the triangle inequality we can see that

$$
\begin{aligned}
& \mid \alpha_{n}(x)-\alpha_{n}(y) \mid \\
& \leq \frac{\sqrt{n}}{n}\left|\sum_{j=1}^{n}\left[K\left(\frac{x-X_{j}}{h_{n, j}}\right)-K\left(\frac{y-X_{j}}{h_{n, j}}\right)\right]\right| \\
&+\sqrt{n}\left(\left|E \widetilde{F}_{n}(x)-F(x)\right|+\left|E \widetilde{F}_{n}(y)-F(y)\right|\right. \\
&+|F(x)-F(y)|) .
\end{aligned}
$$


Since $K$ is a nondecreasing function and applying triangle inequality again we get

$$
\begin{aligned}
& \left|\alpha_{n}(x)-\alpha_{n}(y)\right| \\
& \leq\left|\alpha_{n}(y+q)-\alpha_{n}(y)\right|+\sqrt{n}\left|E \widetilde{F}_{n}(y+q)-E \widetilde{F}_{n}(y)\right| \\
& +\sqrt{n}\left(\left|E \widetilde{F}_{n}(x)-F(x)\right|+\left|E \widetilde{F}_{n}(y)-F(y)\right|\right. \\
& \quad+|F(x)-F(y)|) .
\end{aligned}
$$

Cai and Roussas in [1] showed that under assumptions made on the d.f. $F$ and the kernel function $K$, we have

$$
\left|E \widetilde{F}_{n}(x)-F(x)\right|=O\left(h_{n}^{2}\right)
$$

Similarly, by Taylor expansion, it is easy to see that

$$
|F(x)-F(y)| \leq\|f\| \cdot|x-y|
$$

Thus,

$$
\begin{aligned}
\left|\alpha_{n}(x)-\alpha_{n}(y)\right| \leq & \left|\alpha_{n}(y+q)-\alpha_{n}(y)\right| \\
& +C \sqrt{n h_{n}^{4}}+M \sqrt{n} q,
\end{aligned}
$$

where $M=\sup _{x \in[0,1]} f(x)$ and $C=C_{f^{\prime}, K}$ is a positive constant dependent on the functions $F$ and $K$.

Let us recall that $y \leq x \leq y+q, m_{n}=\left\lfloor\delta / r_{n}\right\rfloor$ and $q \geq \epsilon / n$. Taking $q=(\epsilon / \sqrt{n})\left(=r_{n}\right)$ we notice that $m_{n} q=\left\lfloor\delta / r_{n}\right\rfloor r_{n} \leq \delta$, thus when $n \rightarrow \infty$ the interval $\left[y, y+m_{n} q\right]$ coincides with $[y, y+\delta]$. Approaching the aim of Step 4, let us observe that

$$
\begin{aligned}
\sup _{y \leq x \leq y+m_{n} q}\left|\alpha_{n}(x)-\alpha_{n}(y)\right|= & \max _{1 \leq i \leq m_{n}}\left|\alpha_{n}(y+i q)-\alpha_{n}(y)\right| \\
& +\left|\alpha_{n}\left(x_{0}\right)-\alpha_{n}\left(y+i_{\max } q\right)\right|
\end{aligned}
$$

where $x_{0} \in\left[y+i_{0} q, y+\left(i_{0}+1\right) q\right]$ is a point at which the above supremum is attained and

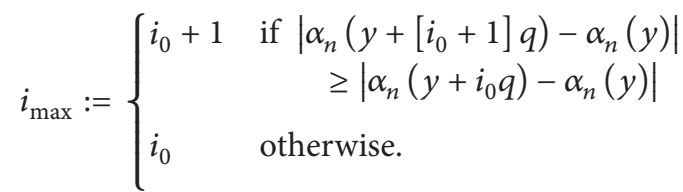

Without the loss of generality, we may assume that $i_{\max }=i_{0}+$ 1, which implies

$$
\begin{array}{r}
\sup _{y \leq x \leq y+m_{n} q}\left|\alpha_{n}(x)-\alpha_{n}(y)\right|=\max _{1 \leq i \leq m_{n}}\left|\alpha_{n}(y+i q)-\alpha_{n}(y)\right| \\
+\left|\alpha_{n}\left(x_{0}\right)-\alpha_{n}\left(y+i_{0} q\right)\right| .
\end{array}
$$

Now, applying inequality (36), the definition of $i_{\max }$ and the triangle inequality we have

$$
\begin{aligned}
\sup _{y \leq x \leq y+m_{n} q}\left|\alpha_{n}(x)-\alpha_{n}(y)\right| \\
\leq \max _{1 \leq i \leq m_{n}}\left|\alpha_{n}(y+i q)-\alpha_{n}(y)\right| \\
\quad+\left|\alpha_{n}\left(y+\left[i_{0}+1\right] q\right)-\alpha_{n}\left(y+i_{0} q\right)\right| \\
\quad+M \sqrt{n} q+C \sqrt{n h_{n}^{4}} \\
\leq \max _{1 \leq i \leq m_{n}}\left|\alpha_{n}(y+i q)-\alpha_{n}(y)\right| \\
\quad+\left|\alpha_{n}\left(y+\left[i_{0}+1\right] q\right)-\alpha_{n}(y)\right| \\
\quad+\left|\alpha_{n}\left(y+i_{0} q\right)-\alpha_{n}(y)\right|+M \sqrt{n} q+C \sqrt{n h_{n}^{4}} \\
\leq 3 \max _{1 \leq i \leq m_{n}}\left|\alpha_{n}(y+i q)-\alpha_{n}(y)\right|+M \sqrt{n} q+C \sqrt{n h_{n}^{4}} .
\end{aligned}
$$

If we plug in $q=r_{n}$ we arrive at

$$
\begin{gathered}
\sup _{y \leq x \leq y+m_{n} r_{n}}\left|\alpha_{n}(x)-\alpha_{n}(y)\right| \leq 3 \max _{1 \leq i \leq m_{n}}\left|\alpha_{n}\left(y+i r_{n}\right)-\alpha_{n}(y)\right| \\
+M \sqrt{n} r_{n}+C \sqrt{n h_{n}^{4}} .
\end{gathered}
$$

Step 5. Finally, we are in a position to obtain inequality (23), that is,

$$
\begin{gathered}
\forall_{\epsilon>0} \forall_{\eta>0} \exists_{0 \leq \delta<1 / 2} \exists_{n_{0}} \forall_{n \geq n_{0}} P\left(\sup _{0 \leq y \leq 1-2 \delta} \sup _{y \leq x \leq y+2 \delta}\left|\alpha_{n}(x)-\alpha_{n}(y)\right|\right. \\
\geq \epsilon) \leq \eta
\end{gathered}
$$

We now successively make use the inequalities (29), (41), (27), (29), and (28) to get

$$
\begin{aligned}
& P\left(\sup _{y \leq x \leq y+2 \delta}\left|\alpha_{n}(x)-\alpha_{n}(y)\right| \geq 4 M \epsilon+C \sqrt{n h_{n}^{4}}\right) \\
& \leq P\left(\sup _{y \leq x \leq y+2\left(m_{n}+1\right) r_{n}}\left|\alpha_{n}(x)-\alpha_{n}(y)\right| \geq 4 M \epsilon+C \sqrt{n h_{n}^{4}}\right) \\
& \leq P\left(3 \max _{1 \leq i \leq 2\left(m_{n}+1\right)}\left|\alpha_{n}\left(y+i r_{n}\right)-\alpha_{n}(y)\right|\right. \\
& \left.+M \sqrt{n} r_{n}+C \sqrt{n h_{n}^{4}} \geq 4 M \epsilon+C \sqrt{n h_{n}^{4}}\right)
\end{aligned}
$$




$$
\begin{aligned}
& =P\left(\max _{1 \leq i \leq 2\left(m_{n}+1\right)}\left|\alpha_{n}\left(y+i r_{n}\right)-\alpha_{n}(y)\right| \geq M \epsilon\right) \\
& \leq \frac{C_{p, \min \left\{p_{1}, p_{2}+p_{3}\right\}}(2 \delta)^{\min \left\{p_{1}, p_{2}+p_{3}\right\}} \leq \eta}{(M \epsilon)^{p}}
\end{aligned}
$$

for any fixed $y \in[0,1]$. Since the upper bound does not depend on $y$ and the probability measure as well as supremum function are continuous, we obtain

$$
P\left(\sup _{0 \leq y \leq 1-2 \delta} \sup _{y \leq x \leq y+2 \delta}\left|\alpha_{n}(x)-\alpha_{n}(y)\right| \geq 4 M \epsilon+C \sqrt{n h_{n}^{4}}\right) \leq \eta,
$$

where $4 M \epsilon+C \sqrt{n h_{n}^{4}}$ is arbitrarily small.

Since condition (10) is checked, the proof is completed.

\section{Tightness of the Standard Empirical Process}

In this section, we deal with the standard empirical process built on an associated sequence of uniformly $[0,1]$ distributed r.v.s $\left\{U_{j}\right\}_{j \geq 1}$, that is,

$$
\alpha_{n}(x)=\frac{1}{\sqrt{n}} \sum_{j=1}^{n}\left(I\left[U_{j} \leq x\right]-x\right),
$$

where $x \in[0,1]$. We shall relax the restrictions imposed on the process by $\mathrm{Yu}$ in [7] to obtain tightness. Precisely, we do not need stationarity any more due to the technique drawn from [14], and we lower the assumed rate at which the covariance tends to zero.

While proving tightness of the empirical process, we will use the criterion proved in the first section as well as some of our covariance inequalities.

We shall start with the fact known under the name of multinomial theorem. We recall it in the following lemma.

Lemma 2. For natural numbers $m, n$ and $x_{1}, x_{2}, \ldots, x_{m} \in \mathbf{R}$

$$
\left(x_{1}+\cdots+x_{n}\right)^{m}=\sum_{k_{1}+\cdots+k_{n}=m}\left(\begin{array}{c}
m \\
k_{1}, \ldots, k_{n}
\end{array}\right) \prod_{1 \leq i \leq n} x_{i}^{k_{i}},
$$

where $\left(\begin{array}{c}m \\ k_{1}, \ldots, k_{n}\end{array}\right)=m ! /\left(k_{1} ! \cdots k_{n} !\right)$ and $k_{1}, \ldots, k_{n} \in$ $\{0,1, \ldots, m\}$.

In particular,

$$
\left(x_{1}+\cdots+x_{n}\right)^{4} \leq 4 ! \cdot \sum_{k_{1}+\cdots+k_{n}=4} x_{1}^{k_{1}} \cdots x_{n}^{k_{n}},
$$

which implies for $S_{n}:=\sum_{i=1}^{n} X_{i}$, that

$$
\begin{aligned}
E S_{n}^{4} & \leq 4 ! \cdot \sum_{k_{1}+\cdots+k_{n}=4} E\left(X_{1}^{k_{1}} \cdots X_{n}^{k_{n}}\right) \\
& \leq 4 ! \cdot \sum_{t=1}^{n} \sum_{0 \leq i, j, k \leq n-1}\left|E\left(X_{t} X_{t+i} X_{t+i+j} X_{t+i+j+k}\right)\right| .
\end{aligned}
$$

Let us now introduce the following notation due to Doukhan and Louhichi (see [14]) for a sequence of centered r.v.s $\left\{X_{t_{n}}\right\}_{n \geq 1}$.

$$
C_{r, q}:=\sup \left|\operatorname{Cov}\left(X_{t_{1}} \cdots X_{t_{m}} ; X_{t_{m}+r} \cdots X_{t_{q}}\right)\right|
$$

where the supremum runs over all divisions of the group composed of $q$ r.v.s into two subgroups, such that the distance between the highest index of the r.v.s in the first group and the lowest index of the r.v.s from the second group is equal to $r$, $r \in\{1,2, \ldots\}$. For $r=0$, we shall define $C_{0, q}=1$. Let us then put

$$
X_{t_{1}}:=I\left[U_{t_{1}} \leq x\right]-x, \quad X_{t_{2}}:=I\left[U_{t_{2}} \leq x\right]-x
$$

We will now estimate the summands in (48).

If $\max \{i, j, k\}=i$, then

$$
\begin{aligned}
& \left|E\left\{X_{t} \cdot\left(X_{t+i} X_{t+i+j} X_{t+i+j+k}\right)\right\}\right| \\
& \quad=\left|\operatorname{Cov}\left(X_{t} ; X_{t+i} X_{t+i+j} X_{t+i+j+k}\right)\right| \leq C_{i, 4},
\end{aligned}
$$

Similarly, for $\max \{i, j, k\}=k$, we have

$$
\begin{aligned}
& \left|E\left\{\left(X_{t} X_{t+i} X_{t+i+j}\right) \cdot X_{t+i+j+k}\right\}\right| \\
& \quad=\left|\operatorname{Cov}\left(X_{t} X_{t+i} X_{t+i+j} ; X_{t+i+j+k}\right)\right| \leq C_{k, 4} .
\end{aligned}
$$

When $\max \{i, j, k\}=j$, then

$$
\begin{aligned}
& \left|E\left\{\left(X_{t} X_{t+i}\right) \cdot\left(X_{t+i+j} X_{t+i+j+k}\right)\right\}\right| \\
& =\mid \operatorname{Cov}\left(X_{t} X_{t+i} ; X_{t+i+j} X_{t+i+j+k}\right) \\
& \quad+E\left(X_{t} X_{t+i}\right) E\left(X_{t+i+j} X_{t+i+j+k}\right) \mid \\
& \leq C_{j, 4}+C_{i, 2} C_{k, 2} .
\end{aligned}
$$

We keep on estimating the fourth moment of $S_{n}$ by introducing $\bar{t} \in\{0, \ldots, n\}$ - the index, for which $\mid E\left(X_{t} X_{t+i} X_{t+i+j}\right.$ $\left.X_{t+i+j+k}\right) \mid$ attains its maximum.

$$
\begin{aligned}
E S_{n}^{4} & \leq 4 ! n \sum_{0 \leq i, j, k \leq n-1}\left|E\left(X_{\bar{t}} X_{\bar{t}+i} X_{\bar{t}+i+j} X_{\bar{t}+i+j+k}\right)\right| \\
& =4 ! n\left(\sum_{0 \leq i, k \leq j}\left[C_{i, 2} \cdot C_{k, 2}+C_{j, 4}\right]+\sum_{0 \leq i, j \leq k} C_{k, 4}+\sum_{0 \leq k, j \leq i} C_{i, 4}\right) \\
& =4 ! n\left(\sum_{0 \leq i, k \leq j} C_{i, 2} \cdot C_{k, 2}+3 \sum_{0 \leq k, j \leq i} C_{i, 4}\right) .
\end{aligned}
$$


The terms obtained in (54) may further be bounded from above in the following way:

$$
\begin{aligned}
\sum_{0 \leq i, k \leq j} C_{i, 2} \cdot C_{k, 2} & =\sum_{j=0}^{n-1} \sum_{i, k=0}^{j} C_{i, 2} C_{k, 2} \\
& \leq n \sum_{i, k=0}^{n-1} C_{i, 2} C_{k, 2}=n\left(\sum_{i=0}^{n-1} C_{i, 2}\right)^{2} \\
\sum_{0 \leq k, j \leq i} C_{i, 4} & =\sum_{i=0}^{n-1} \sum_{j, k=0}^{i} C_{i, 4} \leq \sum_{i=0}^{n-1}(i+1)(i+1) C_{i, 4} \\
& =\sum_{i=0}^{n-1}(i+1)^{2} C_{i, 4}
\end{aligned}
$$

We thus get the inequality

$$
E S_{n}^{4} \leq 4 !\left(\left[n \sum_{i=0}^{n-1} C_{i, 2}\right]^{2}+3 n \sum_{i=0}^{n-1}(i+1)^{2} C_{i, 4}\right) .
$$

We shall now focus on estimating $C_{r, 2}$ and $C_{r, 4}$. Since $U_{t_{1}}$ and $U_{t_{2}}$ are associated uniformly $[0,1]$ distributed r.v.s, $X_{t_{1}}$ and $X_{t_{2}}$ - as monotone functions of these r.v.s - are associated as well. In order to bound

$$
C_{r, 2}=\sup _{0 \leq t_{1}<t_{2} \leq n: t_{2}-t_{1}=r} \operatorname{Cov}\left(X_{t_{1}}, X_{t_{2}}\right),
$$

let us notice that from Schwarz inequality

$$
\begin{aligned}
\operatorname{Cov}\left(X_{t_{1}}, X_{t_{2}}\right) & =E\left(I\left[U_{t_{1}} \leq x\right] I\left[U_{t_{2}} \leq x\right]\right)-x^{2} \\
& \leq \sqrt{x^{2}}-x^{2} \leq x
\end{aligned}
$$

On the other hand, invoking inequalities from $[13,15]$,

$$
\begin{aligned}
& \operatorname{Cov}\left(X_{t_{1}}, X_{t_{2}}\right) \\
& \leq \sup _{x \in[0,1]}\left[P\left(U_{t_{1}} \leq x, U_{t_{2}} \leq x\right)-P\left(U_{t_{1}} \leq x\right) P\left(U_{t_{2}} \leq x\right)\right] \\
& \quad \leq \begin{cases}C \cdot \operatorname{Cov}^{1 / 3}\left(U_{t_{1}}, U_{t_{2}}\right) & \text { for associated r.v.'s } \\
4 \cdot \operatorname{Cov}\left(U_{t_{1}}, U_{t_{2}}\right) & \text { for } \mathrm{MTP}_{2} \text { r.v.'s. }\end{cases}
\end{aligned}
$$

As a consequence,

$$
\begin{aligned}
& C_{r, 2} \\
& \leq \begin{cases}\min \left\{x, C \cdot \operatorname{Cov}^{1 / 3}\left(U_{t_{1}}, U_{t_{2}}\right)\right\} & \text { for associated r.v.'s } \\
\min \left\{x, 4 \cdot \operatorname{Cov}\left(U_{t_{1}}, U_{t_{2}}\right)\right\} & \text { for } \mathrm{MTP}_{2} \text { r.v.'s, }\end{cases}
\end{aligned}
$$

where $t_{2}-t_{1}=r$. Still, we need the upper bound for $C_{r, 4}$. It turns out that

$$
\begin{aligned}
& C_{r, 4} \\
& =\sup \left|\operatorname{Cov}\left\{\prod_{i=1}^{2}\left(I\left[U_{t_{i}} \leq x\right]-x\right) ; \prod_{i=3}^{4}\left(I\left[U_{t_{i}} \leq x\right]-x\right)\right\}\right| .
\end{aligned}
$$

In other words, among all divisions of the group $\left\{X_{t_{i}}, i \in\right.$ $\{1,2,3,4\}\}$ into two subgroups, $C_{r, 4}$ attains the biggest value in case we take two subgroups consisted of two r.v.s. The supremum runs over the set $\left\{t_{1}, t_{2}, t_{3}, t_{4} \in \mathbf{N}: 0 \leq t_{1}<t_{2}<\right.$ $\left.t_{3}<t_{4} \leq n \wedge t_{3}-t_{2}=r\right\}$. Elementary calculation leads to the following formula:

$$
\begin{aligned}
& C_{r, 4}=\sup \mid P\left(U_{t_{i}} \leq x, i \in\{1,2,3,4\}\right) \\
& -P\left(U_{t_{i}} \leq x, i \in\{1,2\}\right) P\left(U_{t_{i}} \leq x, i \in\{3,4\}\right) \\
& -x\left[P\left(U_{t_{i}} \leq x, i \in\{1,2,3\}\right)\right. \\
& -P\left(U_{t_{i}} \leq x, i \in\{1,2\}\right) P\left(U_{t_{3}} \leq x\right) \\
& +P\left(U_{t_{i}} \leq x, i \in\{1,3,4\}\right) \\
& -P\left(U_{t_{i}} \leq x, i \in\{3,4\}\right) P\left(U_{t_{1}} \leq x\right) \\
& +P\left(U_{t_{i}} \leq x, i \in\{1,2,4\}\right) \\
& -P\left(U_{t_{i}} \leq x, i \in\{1,2\}\right) P\left(U_{t_{4}} \leq x\right) \\
& +P\left(U_{t_{i}} \leq x, i \in\{2,3,4\}\right) \\
& \left.-P\left(U_{t_{i}} \leq x, i \in\{3,4\}\right) P\left(U_{t_{2}} \leq x\right)\right] \\
& +x^{2}\left[P\left(U_{t_{1}} \leq x, U_{t_{3}} \leq x\right)\right. \\
& -P\left(U_{t_{1}} \leq x\right) P\left(U_{t_{3}} \leq x\right) \\
& +P\left(U_{t_{1}} \leq x, U_{t_{4}} \leq x\right) \\
& -P\left(U_{t_{1}} \leq x\right) P\left(U_{t_{4}} \leq x\right) \\
& +P\left(U_{t_{2}} \leq x, U_{t_{3}} \leq x\right) \\
& -P\left(U_{t_{2}} \leq x\right) P\left(U_{t_{3}} \leq x\right) \\
& +P\left(U_{t_{2}} \leq x, U_{t_{4}} \leq x\right) \\
& \left.-P\left(U_{t_{2}} \leq x\right) P\left(U_{t_{2}} \leq x\right)\right] \mid \\
& \leq\left|R_{1}\right|+\left|R_{2}\right|+\left|R_{3}\right|,
\end{aligned}
$$

where for the sake of simplicity, $R_{1}, R_{2}$, and $R_{3}$ are, respectively, the free coefficient, the expression with $x$, and the expression with $x^{2}$. Using the Lebowitz inequality (see [15] for instance) and inequalities obtained in $[13,15]$ we arrive at

$$
\begin{aligned}
\left|R_{1}\right|= & \mid P\left(U_{t_{i}} \leq x, i \in\{1,2,3,4\}\right) \\
& \quad-P\left(U_{t_{i}} \leq x, i \in\{1,2\}\right) P\left(U_{t_{i}} \leq x, i \in\{3,4\}\right) \mid \\
\leq & H_{U_{t_{1}}, U_{t_{3}}}+H_{U_{t_{1}}, U_{t_{4}}}+H_{U_{t_{2}}, U_{t_{3}}}+H_{U_{t_{2}}, U_{t_{4}}}
\end{aligned}
$$




$$
\leq \begin{cases}C\left(\sum_{i=1}^{2} \sum_{j=3}^{4} \operatorname{Cov}^{1 / 3}\left(U_{t_{i}}, U_{t_{j}}\right)\right) & \text { for associated r.v.s } \\ 4\left(\sum_{i=1}^{2} \sum_{j=3}^{4} \operatorname{Cov}\left(U_{t_{i}}, U_{t_{j}}\right)\right) & \text { for } \mathrm{MTP}_{2} \text { r.v.s. }\end{cases}
$$

Analogously, we get

$$
\begin{aligned}
& \left|R_{2}\right| \\
& \leq \begin{cases}2 x \cdot C\left(\sum_{i=1}^{2} \sum_{j=3}^{4} \operatorname{Cov}^{1 / 3}\left(U_{t_{i}}, U_{t_{j}}\right)\right) & \text { for associated r.v.s } \\
2 x \cdot 4\left(\sum_{i=1}^{2} \sum_{j=3}^{4} \operatorname{Cov}\left(U_{t_{i}}, U_{t_{j}}\right)\right) & \text { for } \mathrm{MTP}_{2} \text { r.v.'s, }\end{cases} \\
& \left|R_{3}\right| \\
& \leq \begin{cases}x^{2} \cdot C\left(\sum_{i=1}^{2} \sum_{j=3}^{4} \operatorname{Cov}^{1 / 3}\left(U_{t_{i}}, U_{t_{j}}\right)\right) & \text { for associated r.v.'s } \\
x^{2} \cdot 4\left(\sum_{i=1}^{2} \sum_{j=3}^{4} \operatorname{Cov}\left(U_{t_{i}}, U_{t_{j}}\right)\right) & \text { for } \mathrm{MTP}_{2} \text { r.v.'s. }\end{cases}
\end{aligned}
$$

Eventually, we have

$$
\begin{aligned}
& C_{r, 4} \\
& \leq \begin{cases}f(x) \cdot C\left(\sum_{i=1}^{2} \sum_{j=3}^{4} \operatorname{Cov}^{1 / 3}\left(U_{t_{i}}, U_{t_{j}}\right)\right) & \text { for associated r.v.'s } \\
f(x) \cdot 4\left(\sum_{i=1}^{2} \sum_{j=3}^{4} \operatorname{Cov}\left(U_{t_{i}}, U_{t_{j}}\right)\right) & \text { for } \mathrm{MTP}_{2} \text { r.v.'s, }\end{cases}
\end{aligned}
$$

where $t_{3}-t_{2}=r$ and $f(x)=\left(1+2 x+x^{2}\right)$ for $x \in[0,1]$.

Let us now introduce the following notation:

$$
\theta_{r}= \begin{cases}\sup _{t \in \mathbf{N}} \operatorname{Cov}\left(U_{t}, U_{t+r}\right) & \text { for } r \in\{1,2, \ldots\} \\ 1 & \text { for } r=0\end{cases}
$$

and assume it decays powerly at rate $a$ in the following way:

$$
\theta_{r}=O\left(\frac{1}{(r+1)^{a}}\right) \quad \text { for } a>0, r \in\{0,1, \ldots\}
$$

Let us get back to inequality (56), we can now carry on. At first, for associated r.v.s,

$$
\begin{aligned}
E S_{n}^{4} \leq & 4 !\left[n \sum_{r=0}^{n-1} \min \left\{x, C \cdot \operatorname{Cov}^{1 / 3}\left(U_{t_{1}}, U_{t_{2}}\right)\right\}\right]^{2} \\
+ & 4 ! \cdot 3 n \sum_{r=0}^{n-1}(r+1)^{2} f(x) \cdot C\left(\sum_{i=1}^{2} \sum_{j=3}^{4} \operatorname{Cov}^{1 / 3}\left(U_{t_{i}}, U_{t_{j}}\right)\right) \\
\leq & D \cdot\left(\left[n \sum_{r=0}^{n-1} \min \left\{x,(r+1)^{-a / 3}\right\}\right]^{2}\right. \\
& \left.+n \sum_{r=0}^{n-1}(r+1)^{2}(r+1)^{-a / 3}\right) \\
= & D \cdot\left(\left[n \sum_{r=1}^{n} \min \left\{x, r^{-a / 3}\right\}\right]^{2}+n \sum_{r=1}^{n} r^{2} r^{-a / 3}\right) \\
\leq & D \cdot\left(\left[n \sum_{r<x^{-3 / a}} x+n \sum_{r \geq x^{-3 / a}} \frac{1}{r^{a / 3}}\right]^{2}+n \sum_{r=1}^{n} r^{2-a / 3}\right) \\
\leq & D_{1} \cdot\left(n^{2} x^{2(a-3) / a}+\xi\right),
\end{aligned}
$$

where $D$ and $D_{1}$ are constants and

$$
\xi=n \sum_{r=1}^{n} r^{2-a / 3}= \begin{cases}O(n), & a>9 \\ O(n \ln n), & a=9 \\ O\left(n^{4-a / 3}\right), & 3<a<9 .\end{cases}
$$

It is worth mentioning, that in the last inequality of (68), we used the estimate

$$
\int_{x}^{\infty} \frac{1}{t^{p}} d t \sim \frac{1}{x^{p-1}} \quad \text { for } p>1
$$

At the same time, in the case of $\mathrm{MTP}_{2}$ r.v.s, we get

$$
E S_{n}^{4} \leq D_{2} \cdot\left(n^{2} x^{2(a-1) / a}+\zeta\right),
$$

where $D_{2}$ is constant and

$$
\zeta=n \sum_{r=1}^{n} r^{2-a}= \begin{cases}O(n), & a>3 \\ O(n \ln n), & a=3 \\ O\left(n^{4-a}\right), & 1<a<3 .\end{cases}
$$

Let now $\alpha_{n}(x)=(1 / \sqrt{n}) \sum_{i=1}^{n}\left(I\left[U_{t_{i}} \leq x\right]-x\right)$. Then

$$
\alpha_{n}(x)-\alpha_{n}(y)=\frac{1}{\sqrt{n}} \sum_{i=1}^{n}\left(I\left[x<U_{t_{i}} \leq y\right]-(x-y)\right)
$$


has the fourth moment estimated-in the case of associated r.v.s-by

$$
\begin{aligned}
E\left[\alpha_{n}(x)-\alpha_{n}(y)\right]^{4} & \\
= & E\left[\frac{1}{\sqrt{n}} \sum_{i=1}^{n}\left(I\left[x<U_{t_{i}} \leq y\right]-(x-y)\right)\right]^{4} \\
= & \frac{1}{n^{2}} E\left[\sum_{i=1}^{n}\left(I\left[x<U_{t_{i}} \leq y\right]-(x-y)\right)\right]^{4} \\
\leq & D_{1} \cdot\left(\frac{1}{n^{2}} n^{2}|x-y|^{2(a-3) / a}+\frac{\xi}{n^{2}}\right) \\
= & D_{1} \cdot\left(|x-y|^{2(a-3) / a}+\frac{\xi}{n^{2}}\right) \\
= & \left\{\begin{array}{l}
O\left(n^{-1}\right), \quad a>9 \\
O\left(\frac{\ln n}{n}\right), \quad a=9 \\
O\left(n^{2-a / 3}\right), \quad 3<a<9
\end{array}\right.
\end{aligned}
$$

and in the case of $\mathrm{MTP}_{2}$ r.v.s by

$$
\begin{aligned}
E\left[\alpha_{n}(x)-\alpha_{n}(y)\right]^{4} \leq & D_{2} \cdot\left(|x-y|^{2(a-1) / a}+\frac{\zeta}{n^{2}}\right) \\
& = \begin{cases}O\left(n^{-1}\right), & a>3 \\
O\left(\frac{\ln n}{n}\right), & a=3 \\
O\left(n^{2-a}\right), & 1<a<3 .\end{cases}
\end{aligned}
$$

In light of the Shao and Yu's criterion, our process is tight for associated r.v.s when $a>6$ and for MTP 2 r.v.s when $a>2$. Let us sum up this result in the following theorem.

Theorem 3. Let $\alpha_{n}(x)=(1 / \sqrt{n}) \sum_{i=1}^{n}\left(I\left[U_{i} \leq x\right]-x\right)$ be the empirical process built on an associated sequence of uniformly $[0,1]$ distributed r.v.s $\left\{U_{i}\right\}_{i \geq 1}$. Let also

$$
\begin{array}{r}
\theta_{r}:=\sup _{t \in \mathbf{N}} \operatorname{Cov}\left(U_{t}, U_{t+r}\right)=O\left((r+1)^{-a}\right), \\
\text { where } r \in\{0,1, \ldots\}, a>0 .
\end{array}
$$

Then $\left\{\alpha_{n}(x)\right\}_{n \geq 1}$ is tight for $a>6$. If the r.v.s $\left\{U_{i}\right\}_{i \geq 1}$ are $M T P_{2}$, then the process is tight for $a>2$.

$\mathrm{Yu}$ assumed stationarity of $\left\{U_{i}\right\}_{i>1}$ and $\sum_{n=1}^{\infty} n^{6,5+v} \operatorname{Cov}\left(U_{0}, U_{n}\right)<\infty$ for a positive constant $v$, thus, the rate of decay $a>7,5$. Our result weakens considerably these assumptions especially in the case of $\mathrm{MTP}_{2}$ r.v.s.

Louhichi, in [16], proposed a different tightness criterion involving the so-called bracketing numbers. She managed to enhance Yu's result-even more than Shao and Yu in [8]-since she proved that it suffices to take $a>4$ to get tightness of the empirical process based on the associated r.v.s. Nevertheless, she kept the assumption of stationarity valid.

In the final analysis, our result's advantage is the absence of the stationarity assumption and the rate of decay for $\theta_{r}$ remains (up to the author's knowledge) unimproved for $\mathrm{MTP}_{2}$ r.v.s.

Unfortunately, with a view to obtaining weak convergence of the process in question, that is also convergence of finite-dimensional distributions, we do not know how to manage without the assumption of stationarity. Therefore, we conclude with the following corollary.

Corollary 4. Let $\alpha_{n}(x)=(1 / \sqrt{n}) \sum_{i=1}^{n}\left(I\left[U_{i} \leq x\right]-x\right)$ be the empirical process built on a stationary associated sequence of uniformly $[0,1]$ distributed r.v.s $\left\{U_{i}\right\}_{i \geq 1}$. Let also

$$
\begin{gathered}
\theta_{r}:=\operatorname{Cov}\left(U_{1}, U_{1+r}\right)=O\left((r+1)^{-a}\right), \\
\text { where } r \in\{0,1, \ldots\}, a>0 .
\end{gathered}
$$

Then, if $a>6$

$$
\alpha_{n}(\cdot) \longrightarrow B(\cdot) \quad \text { weakly in } D[0,1],
$$

where $B(\cdot)$ is the zero mean Gaussian process on $[0,1]$ with covariance structure defined by

$$
\begin{aligned}
& \sigma^{2}(x, y)=x \wedge y-x y \\
&+\sum_{j=1}^{\infty}\left[\operatorname{Cov}\left(I\left[U_{1} \leq x\right], I\left[U_{j+1} \leq y\right]\right)\right. \\
& \\
&\left.\quad+\operatorname{Cov}\left(I\left[U_{1} \leq y\right], I\left[U_{j+1} \leq x\right]\right)\right] .
\end{aligned}
$$

If the r.v.s $\left\{U_{i}\right\}_{i \geq 1}$ are $M T P_{2}$, then it suffices to be $a>2$ in order to claim the above convergence.

Proof. It remains to establish convergence of finite-dimensional distributions repeating the procedure from [7].

\section{Tightness of the Kernel-Type Empirical Process}

In this section we shall weaken assumption imposed on the covariance structure of r.v.s $\left\{X_{j}\right\}_{j \geq 1}$ by Cai and Roussas in [1] for the kernel estimator of the d.f.

$$
\bar{F}_{n}(x)=\frac{1}{n} \sum_{j=1}^{n} K\left(\frac{x-X_{j}}{h_{n}}\right) .
$$

They deal with a stationary sequence of negatively associated r.v.s (c.f. [17]) and need the same condition as $\mathrm{Yu}$ [7], that is,

$$
\left|\operatorname{Cov}\left(X_{1}, X_{1+n}\right)\right|=O\left(\frac{1}{n^{7,5+v}}\right)
$$

to get tightness of the smooth empirical process (see condition (A4) in [1]). 
It turns out that it suffices to have

$$
\left|\operatorname{Cov}\left(U_{1}, U_{1+n}\right)\right|=O\left(\frac{1}{n^{3 p /(p-1)}}\right)
$$

where $p>4$ is a positive constant taken from the tightness criterion (8). It is easy to see that asymptotically we get the rate 3.

On the way to prove it, we will also take use of a Rosenthal-type inequality due to Shao and Yu (see Theorem 2 in [8]) we shall recall in the following lemma.

Lemma 5. Let $p>2$ and $f$ be a real valued function bounded by 1 with bounded first derivative. Suppose that $\left\{X_{n}\right\}_{n \geq 1}$ is a sequence of stationary and associated r.v.s, such that for $n \in \mathbf{N}$

$$
\operatorname{Cov}\left(X_{1}, X_{n}\right)=O\left(n^{-b}\right), \quad \text { for some } b>p-1 \text {. }
$$

Then, for any $\mu>0$ there exists some positive constant $k_{\mu}$ independent of the function $f$, for which

$$
\begin{aligned}
& E\left|S_{n}(f)-E S_{n}(f)\right|^{p} \\
& \quad \leq k_{\mu}\left(n^{1+\mu}\left\|f^{\prime}\right\|^{2}+n^{p / 2}\left[\sum_{j=1}^{n}\left|\operatorname{Cov}\left(f\left(X_{1}\right), f\left(X_{j}\right)\right)\right|\right]\right) .
\end{aligned}
$$

As we can see, the lemma assumes association, but it works for negatively associated r.v.'s as well, since in the proof, it reaches back the result of Newman (see Proposition 15 in [18]), where both types of association are allowed.

Let us recall that $\alpha_{n}(x)=\sqrt{n}\left(\bar{F}_{n}(x)-E \bar{F}_{n}(x)\right)$, where $\bar{F}_{n}(x)=(1 / n) \sum_{j=1}^{n} K\left(\left(x-X_{j}\right) / h_{n}\right)$. It is easy to see that

$$
E\left|\alpha_{n}(x)-\alpha_{n}(y)\right|^{p}=n^{-p / 2} E\left|S_{n}\left(f\left(X_{j}\right)\right)-E S_{n}\left(f\left(X_{j}\right)\right)\right|^{p},
$$

where

$$
\begin{gathered}
f\left(X_{j}\right)=K\left(\frac{x-X_{j}}{h_{n}}\right)-K\left(\frac{y-X_{j}}{h_{n}}\right), \\
S_{n}(f)=\sum_{j=1}^{n} f\left(X_{j}\right) .
\end{gathered}
$$

With an intent to use Lemma 5, we need $f$ to be bounded from above by 1 (which in our case is obvious) and to have bounded $f^{\prime}$; thus, we assume

$$
\sup _{t \in \mathbf{R}}\left|K^{\prime}(t)\right| \frac{1}{h_{n}} \leq \frac{C_{K}}{h_{n}}<\infty .
$$

Now, applying inequality (84) we have

$$
\begin{aligned}
& E\left|\alpha_{n}(x)-\alpha_{n}(y)\right|^{p} \\
& \leq k_{\mu} n^{-p / 2}\left\{n^{1+\mu} \frac{C_{K}^{2}}{h_{n}^{2}}+n^{p / 2}\left[\sum_{j=1}^{n}\left|\operatorname{Cov}\left(f\left(X_{1}\right), f\left(X_{j}\right)\right)\right|\right]^{p / 2}\right\} .
\end{aligned}
$$

Newman showed in [18] that

$$
\begin{aligned}
& \operatorname{Cov}\left(f_{1}(X), f_{2}(Y)\right) \\
& =\iint_{\mathbf{R}^{2}} f_{1}^{\prime}(x) f_{2}^{\prime}(y) \\
& \quad \times[P(X \leq x, Y \leq y)-P(X \leq x) P(Y \leq y)] d x d y
\end{aligned}
$$

if $f_{1}$ and $f_{2}$ are real valued functions on $\mathbf{R}$ having square integrable derivatives $f_{1}^{\prime}$ and $f_{2}^{\prime}$, respectively, and provided that $f_{1}(X), f_{2}(Y)$ have finite second moments. In light of that equation and linearity of covariance, we get

$$
\begin{aligned}
\left|\operatorname{Cov}\left(f\left(X_{1}\right), f\left(X_{j}\right)\right)\right| & \\
=\mid \iint_{\mathbf{R}^{2}}\{[ & P\left(X_{1}<x-h_{n} s, X_{j}<x-h_{n} t\right) \\
- & \left.P\left(X_{1}<x-h_{n} s, X_{j}<y-h_{n} t\right)\right] \\
- & {\left[P\left(X_{1}<x-h_{n} s\right) P\left(X_{j}<x-h_{n} t\right)\right.} \\
- & \left.P\left(X_{1}<x-h_{n} s\right) P\left(X_{j}<y-h_{n} t\right)\right] \\
- & P\left(X_{1}<y-h_{n} s, X_{j}<x-h_{n} t\right) \\
- & \left.P\left(X_{1}<y-h_{n} s, X_{j}<y-h_{n} t\right)\right] \\
- & {\left[P\left(X_{1}<y-h_{n} s\right) P\left(X_{j}<y-h_{n} t\right)\right.} \\
& -P\left(X_{1}<y-h_{n} s\right) \\
& \left.\left.\times P\left(X_{j}<x-h_{n} t\right)\right]\right\} k(s) k(t) d s d t \mid .
\end{aligned}
$$

Without the loss of generality, we may and do assume that $x>y$, thus

$$
\begin{aligned}
\left|\operatorname{Cov}\left(f\left(X_{1}\right), f\left(X_{j}\right)\right)\right| \\
=\mid \iint_{\mathbf{R}^{2}}\left\{P \left(y-h_{n} s \leq X_{1}<x-h_{n} s\right.\right. \\
\left.y-h_{n} t \leq X_{j}<x-h_{n} t\right) \\
-P\left(y-h_{n} s \leq X_{1}<x-h_{n} s\right) \\
\left.\times P\left(y-h_{n} t \leq X_{j}<x-h_{n} t\right)\right\} k(s) k(t) d s d t \mid
\end{aligned}
$$

and by triangle inequality we get

$$
\begin{aligned}
&\left|\operatorname{Cov}\left(f\left(X_{1}\right), f\left(X_{j}\right)\right)\right| \\
& \leq \iint_{\mathbf{R}^{2}} {\left[\int_{y-h_{n} s}^{x-h_{n} s} \int_{y-h_{n} t}^{x-h_{n} t} f_{X_{1}, X_{j}}(u, v) d u d v\right.} \\
&\left.+\int_{y-h_{n} s}^{x-h_{n} s} f_{X_{1}}(u) d u \int_{y-h_{n} t}^{x-h_{n} t} f_{X_{j}}(v) d v\right] k(s) k(t) d s d t .
\end{aligned}
$$


$f_{X_{1}, X_{j}}(u, v), f_{X_{1}}(u)$ and $f_{X_{j}}(v)$ are the joint p.d.f. of $\left[X_{1}, X_{j}\right]$ and marginal p.d.f.'s of r.v.s $X_{1}$ and $X_{j}$, respectively. We need to further assume that $C_{1}$ stands for a common upper bound of $f_{X_{1}}$ and $f_{X_{1}, X_{j}}$ for all $j \in \mathbf{N}$, that is,

$$
\left\|f_{X_{1}}\right\| \leq C_{1}, \quad\left\|f_{X_{1}, X_{j}}\right\| \leq C_{1} \quad \forall j \geq 1 .
$$

Then, we finally obtain

$$
\begin{gathered}
\left|\operatorname{Cov}\left(f\left(X_{1}\right), f\left(X_{j}\right)\right)\right| \leq 2 \widetilde{C}_{1}|x-y|^{2}, \\
\text { where } \widetilde{M}=\max \left\{C_{1}, C_{1}^{2}\right\} .
\end{gathered}
$$

On the other hand, proceeding like Cai and Roussas in [1], we can shortly get

$$
\begin{aligned}
\mid \operatorname{Cov}( & \left.f\left(X_{1}\right), f\left(X_{j}\right)\right) \mid \\
\leq & \operatorname{Cov}\left(K\left(\frac{x-X_{1}}{h_{n}}\right), K\left(\frac{x-X_{j}}{h_{n}}\right)\right) \mid \\
& +\left|\operatorname{Cov}\left(K\left(\frac{x-X_{1}}{h_{n}}\right), K\left(\frac{y-X_{j}}{h_{n}}\right)\right)\right| \\
& +\left|\operatorname{Cov}\left(K\left(\frac{y-X_{1}}{h_{n}}\right), K\left(\frac{x-X_{j}}{h_{n}}\right)\right)\right| \\
& +\left|\operatorname{Cov}\left(K\left(\frac{y-X_{1}}{h_{n}}\right), K\left(\frac{y-X_{j}}{h_{n}}\right)\right)\right| \\
\leq & 4 C_{2}\left|\operatorname{Cov}\left(X_{1}, X_{j}\right)\right|^{1 / 3},
\end{aligned}
$$

where $C_{2}$ is a constant relevant to the covariance inequality for negatively associated r.v.s (see [13]).

We now arrive at the following inequality:

$$
\begin{aligned}
& \left|\operatorname{Cov}\left(f\left(X_{1}\right), f\left(X_{j}\right)\right)\right| \\
& \quad \leq \max \left\{C_{1}, C_{2}\right\}\left\{|x-y|^{2},\left|\operatorname{Cov}\left(X_{1}, X_{j}\right)\right|^{1 / 3}\right\} .
\end{aligned}
$$

Assuming $\left|\operatorname{Cov}\left(f\left(X_{1}\right), f\left(X_{j}\right)\right)\right|=O\left(j^{-r}\right)$ and proceeding similarly to (68), we can get

$$
\begin{aligned}
& {\left[\sum_{j=1}^{n}\left|\operatorname{Cov}\left(f\left(X_{1}\right), f\left(X_{j}\right)\right)\right|^{p / 2}\right.} \\
& \leq \max \left\{C_{1}, C_{2}\right\}\left[\sum_{j<|x-y|^{-2 /(r / 3)}}|x-y|^{2}+\sum_{j \geq|x-y|^{-2 /(r / 3)}} \frac{1}{j^{r / 3}}\right]^{p / 2} \\
& \leq 2 \max \left\{C_{1}, C_{2}\right\}|x-y|^{((r-3) / r) p .}
\end{aligned}
$$

To sum up, we obtain the following inequality:

$$
\begin{aligned}
& E\left|\alpha_{n}(x)-\alpha_{n}(y)\right|^{p} \\
& \quad \leq k_{\mu}\left\{n^{1+\mu-p / 2} \frac{C_{K}^{2}}{h_{n}^{2}}+2 \max \left\{C_{1}, C_{2}\right\}|x-y|^{((r-3) / r) p}\right\} \\
& \quad \leq \widetilde{C}\left\{n^{1+\mu-p / 2} \frac{1}{h_{n}^{2}}+|x-y|^{((r-3) / r) p}\right\},
\end{aligned}
$$

where $\widetilde{C}=k_{\mu} \max \left\{C_{k}^{2}, 2 \max \left\{C_{1}, C_{2}\right\}\right\}$. The formula of the tightness criterion (8) implies that $((r-3) / r) p>1$, so

$$
r>\frac{3 p}{p-1}, \quad \text { where } p>2 .
$$

Let us recall the assumptions imposed on the bandwidhts $\left\{h_{n}\right\}_{n \geq 1}$ by Cai and Roussas in [1]:

B1: $\lim _{n \rightarrow \infty} h_{n}=0$ and $h_{n}>0$ for all $n \in \mathbf{N}_{+}$

B2: $\lim _{n \rightarrow \infty} n h_{n}=\infty$, thus $h_{n}=O\left(1 / n^{1-\beta}\right), \beta>0$

B3: $\lim _{n \rightarrow \infty} n h_{n}^{4}=0$, hence $h_{n}=O\left(1 / n^{1 / 4+\delta}\right), \delta>0$.

In light of the above,

$$
n^{1+\mu-p / 2} \frac{1}{h_{n}^{2}}=n^{3 / 2+\mu+2 \delta-p / 2},
$$

where $\mu>0, p>2$ and $0<\delta<3 / 4$. Confrontation with the tightness criterion (8), forces

$$
\frac{3}{2}+\mu+2 \delta-\frac{p}{2}<-\frac{1}{2}
$$

which implies $p>4$. Let us conclude with the following theorem.

Theorem 6. Let $\alpha_{n}(x)=\sqrt{n}\left(\bar{F}_{n}(x)-E \bar{F}_{n}(x)\right)$ be the empirical process built on the kernel estimator of the d.f. F for a stationary sequence of negatively associated r.v.s. Assume that conditions $A 1, A 2$, (87), B1, B2, B3 are satisfied. Then, provided that the tightness criterion (8) holds with $p>4$, it suffices to demand

$$
\left|\operatorname{Cov}\left(U_{1}, U_{1+n}\right)\right|=O\left(\frac{1}{n^{3 p /(p-1)}}\right)
$$

in order to obtain

$$
\alpha_{n}(\cdot) \longrightarrow B(\cdot) \quad \text { weakly in } D[0,1],
$$

where $B(\cdot)$ is the zero mean Gaussian process with covariance structure defined by

$$
\begin{aligned}
& \sigma^{2}(x, y)= F(x \wedge y)-F(x) F(y) \\
&+\sum_{j=1}^{\infty}\left[\operatorname{Cov}\left(I\left[X_{1} \leq x\right], I\left[X_{j+1} \leq y\right]\right)\right. \\
&\left.\quad+\operatorname{Cov}\left(I\left[X_{1} \leq y\right], I\left[X_{j+1} \leq x\right]\right)\right],
\end{aligned}
$$

and $x, y \in[0,1]$.

Proof. The remaining covergence of finite-dimensional distributions of $\alpha_{n}(x)$ is established in [1]. 


\section{Weak Convergence of the Recursive Kernel-Type Empirical Process under I.I.D. Assumption}

The aim of this section is to show weak convergence of the empirical process:

$$
\alpha_{n}(x)=\frac{1}{\sqrt{n}} \sum_{j=1}^{n}\left[K\left(\frac{x-X_{j}}{h_{j}}\right)-E K\left(\frac{x-X_{j}}{h_{j}}\right)\right]
$$

built on i.i.d. r.v.s $\left\{X_{j}\right\}_{j \geq 1}$.

We will prove that $\alpha_{n}(\cdot)$ converges weakly to the Brownian bridge $B(\cdot)$ with the following covariance structure

$$
\sigma^{2}(x, y)=F(x \wedge y)-F(x) F(y) \quad \text { for } x, y \in[0,1]
$$

It turns out that the tightness criterion (8) suffices to reach the goal. Convergence of finite-dimensional distributions of $\alpha_{n}(\cdot)$ holds and we shall show it.

Proceeding like Cai and Roussas in [1], we need to show that for any $a, b \in \mathbf{R}$

$$
a \alpha_{n}(x)+b \alpha_{n}(y) \longrightarrow a B(x)+b B(y) \quad \text { in distribution. }
$$

Let us introduce the notation

$$
Y_{i}(x):=K\left(\frac{x-X_{i}}{h_{i}}\right)-E K\left(\frac{x-X_{i}}{h_{i}}\right)
$$

and look into the covariance structure of $\alpha_{n}(\cdot)$

$$
\begin{aligned}
\operatorname{Cov} & \left(\alpha_{n}(x), \alpha_{n}(y)\right) \\
& =\operatorname{Cov}\left(\frac{1}{\sqrt{n}} \sum_{i=1}^{n} Y_{i}(x), \frac{1}{\sqrt{n}} \sum_{i=1}^{n} Y_{i}(y)\right) \\
& =\frac{1}{n} \sum_{i=1}^{n} \operatorname{Cov}\left(Y_{i}(x), Y_{i}(y)\right) \\
& =\frac{1}{n} \sum_{i=1}^{n} \operatorname{Cov}\left(K\left(\frac{x-X_{i}}{h_{i}}\right), K\left(\frac{y-X_{i}}{h_{i}}\right)\right),
\end{aligned}
$$

where the second equality follows from assumed independence of r.v.s $\left\{X_{i}\right\}_{i \geq 1}$.

Firstly, we observe that each summand converges to $F(x \wedge$ $y)-F(x) F(y)$, since

$$
\begin{aligned}
\operatorname{Cov}\left(K\left(\frac{x-X_{i}}{h_{i}}\right), K\left(\frac{y-X_{i}}{h_{i}}\right)\right) \\
=E\left[K\left(\frac{x-X_{i}}{h_{i}}\right) K\left(\frac{y-X_{i}}{h_{i}}\right)\right] \\
-E K\left(\frac{x-X_{i}}{h_{i}}\right) E K\left(\frac{y-X_{i}}{h_{i}}\right),
\end{aligned}
$$

where

$$
\begin{aligned}
E[K & \left.\left(\frac{x-X_{i}}{h_{i}}\right) K\left(\frac{y-X_{i}}{h_{i}}\right)\right] \\
= & \int_{-\infty}^{x \wedge y} K\left(\frac{x-t}{h_{i}}\right) K\left(\frac{y-t}{h_{i}}\right) d F(t) \\
& +\int_{x \wedge y}^{x \vee y} K\left(\frac{x-t}{h_{i}}\right) K\left(\frac{y-t}{h_{i}}\right) d F(t) \\
& +\int_{x \vee y}^{\infty} K\left(\frac{x-t}{h_{i}}\right) K\left(\frac{y-t}{h_{i}}\right) d F(t)
\end{aligned}
$$

and $F$ denotes the common d.f. of the r.v.s $\left\{X_{i}\right\}_{i \geq 1}$. In [6], it was shown that

$$
E K\left(\frac{x-X_{i}}{h_{i}}\right) \longrightarrow F(x)
$$

and recalling that the kernel function $K$ is a d.f. as well, we arrive at the conclusion.

Secondly, applying Toeplitz lemma, we get

$$
\begin{aligned}
\operatorname{Cov}\left(\alpha_{n}(x), \alpha_{n}(y)\right) & \longrightarrow F(x \wedge y)-F(x) F(y) \\
= & : \sigma^{2}(x, y), \quad n \longrightarrow \infty .
\end{aligned}
$$

Thus,

$$
\begin{aligned}
& \operatorname{Var}\left(a \alpha_{n}(x)+b \alpha_{n}(y)\right) \\
& =a^{2} \operatorname{Var}\left(\alpha_{n}(x)\right)+2 a b \operatorname{Cov}\left(\alpha_{n}(x), \alpha_{n}(y)\right) \\
& \quad+b^{2} \operatorname{Var}\left(\alpha_{n}(y)\right) \\
& \longrightarrow a^{2} \sigma^{2}(x, x)+2 a b \sigma^{2}(x, y)+b^{2} \sigma^{2}(y, y), \quad n \rightarrow \infty .
\end{aligned}
$$

We are now on the way to prove that $a \alpha_{n}(x)+b \alpha_{n}(y)$ converges in distribution to $a B(x)+b B(y) \sim \mathcal{N}\left(0, a^{2} \sigma^{2}(x, x)+\right.$ $\left.2 a b \sigma^{2}(x, y)+b^{2} \sigma^{2}(y, y)\right)$, which in other words means that

$$
\frac{1}{\sqrt{n}} \sum_{i=1}^{\infty} \frac{a Y_{i}(x)+b Y_{i}(y)}{\sqrt{\operatorname{Var}\left(a \alpha_{n}(x)+b \alpha_{n}(y)\right)}} \longrightarrow \mathcal{N}(0,1) .
$$

In order to obtain (115), we will use Lyapunov condition

$$
\lim _{n \rightarrow \infty} \frac{1}{D_{n}^{2+\delta}} \sum_{i=1}^{n} E\left|a Y_{i}(x)+b Y_{i}(y)\right|^{2+\delta}=0
$$

for $\delta=1$, where $D_{n}^{2}:=\operatorname{Var}\left(\sum_{i=1}^{n}\left[a Y_{i}(x)+b Y_{i}(y)\right]\right)$. Since $|K(\cdot)-E K(\cdot)| \leq 2$,

$$
E\left|a Y_{i}(x)+b Y_{\mathrm{i}}(y)\right|^{3} \leq 8\left(a^{3}+b^{3}\right) .
$$

Moreover, $D_{n}^{2}=n \operatorname{Var}\left(a \alpha_{n}(x)+b \alpha_{n}(y)\right)$ together with (114), yields

$$
D_{n}^{3}=O\left(n^{3 / 2}\right)
$$


Combining (117) with (118), we obtain

$$
\frac{1}{D_{n}^{3}} \sum_{i=1}^{n} E\left|a Y_{i}(x)+b Y_{i}(y)\right|^{3}=O\left(n^{-1 / 2}\right),
$$

which shows that Lyapunov condition holds and completes the proof of convergence of finite-dimensional distributions of $\alpha_{n}(x)$.

We summarize the result of that section in the following theorem.

Theorem 7. Let $\alpha_{n}(x)$ be the recursive kernel-type empirical process defined by (105) built on i.i.d. r.v.s $\left\{X_{j}\right\}_{j \geq 1}$ with marginal d.f. F. If $\alpha_{n}(\cdot)$ satisfies the tightness criterion (8) then

$$
\alpha_{n}(\cdot) \longrightarrow B(\cdot) \quad \text { weakly in } D[0,1]
$$

where $B$ is the Brownian bridge.

\section{Acknowledgment}

The author would like to thank the referees for the careful reading of the paper and helpful remarks.

\section{References}

[1] Z. Cai and G. G. Roussas, "Weak convergence for smooth estimator of a distribution function under negative association," Stochastic Analysis and Applications, vol. 17, no. 2, pp. 145-168, 1999.

[2] Z. Cai and G. G. Roussas, "Efficient Estimation of a Distribution Function under Qudrant Dependence," Scandinavian Journal of Statistics, vol. 25, no. 1, pp. 211-224, 1998.

[3] Z. W. Cai and G. G. Roussas, "Uniform strong estimation under $\alpha$-mixing, with rates," Statistics \& Probability Letters, vol. 15, no. 1, pp. 47-55, 1992.

[4] G. G. Roussas, "Kernel estimates under association: strong uniform consistency," Statistics \& Probability Letters, vol. 12, no. 5, pp. 393-403, 1991.

[5] Y. F. Li and S. C. Yang, "Uniformly asymptotic normality of the smooth estimation of the distribution function under associated samples," Acta Mathematicae Applicatae Sinica, vol. 28, no. 4, pp. 639-651, 2005.

[6] Y. Li, C. Wei, and S. Yang, "The recursive kernel distribution function estimator based on negatively and positively associated sequences," Communications in Statistics, vol. 39, no. 20, pp. 3585-3595, 2010.

[7] H. Yu, "A Glivenko-Cantelli lemma and weak convergence for empirical processes of associated sequences," Probability Theory and Related Fields, vol. 95, no. 3, pp. 357-370, 1993.

[8] Q. M. Shao and H. Yu, "Weak convergence for weighted empirical processes of dependent sequences," The Annals of Probability, vol. 24, no. 4, pp. 2098-2127, 1996.

[9] P. Billingsley, Convergence of Probability Measures, Wiley Series in Probability and Statistics: Probability and Statistics, John Wiley \& Sons, New York, NY, USA, 1999.

[10] J. D. Esary, F. Proschan, and D. W. Walkup, "Association of random variables, with applications," Annals of Mathematical Statistics, vol. 38, pp. 1466-1474, 1967.
[11] A. Bulinski and A. Shashkin:, Limit Theorems for Associated Random Fields and Related Systems, vol. 10 of Advanced Series on Statistical Science and Applied Probability, World Scientific, 2007.

[12] S. Karlin and Y. Rinott, "Classes of orderings of measures and related correlation inequalities. I. Multivariate totally positive distributions," Journal of Multivariate Analysis, vol. 10, no. 4, pp. 467-498, 1980

[13] P. Matuła and M. Ziemba, "Generalized covariance inequalities," Central European Journal of Mathematics, vol. 9, no. 2, pp. 281-293, 2011.

[14] P. Doukhan and S. Louhichi, "A new weak dependence condition and applications to moment inequalities," Stochastic Processes and their Applications, vol. 84, no. 2, pp. 313-342, 1999.

[15] P. Matuła, "A note on some inequalities for certain classes of positively dependent random variables," Probability and Mathematical Statistics, vol. 24, no. 1, pp. 17-26, 2004.

[16] S. Louhichi, "Weak convergence for empirical processes of associated sequences," Annales de l'Institut Henri Poincaré, vol. 36, no. 5, pp. 547-567, 2000.

[17] K. Joag-Dev and F. Proschan, "Negative association of random variables, with applications," The Annals of Statistics, vol. 11, no. 1, pp. 286-295, 1983.

[18] C. M. Newman, "Asymptotic independence and limit theorems for positively and negatively dependent random variables," in Inequalities in Statistics and Probability, Y. L. Tong, Ed., vol. 5, pp. 127-140, Institute of Mathematical Statistics, Hayward, Calif, USA, 1984. 


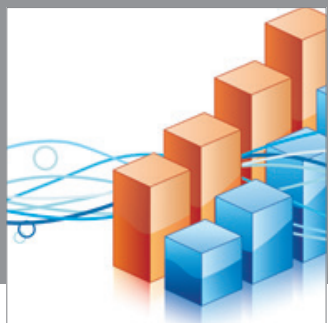

Advances in

Operations Research

mansans

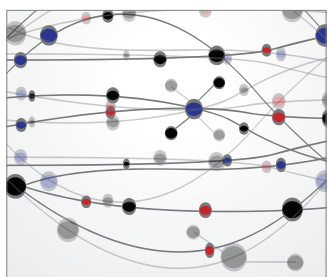

The Scientific World Journal
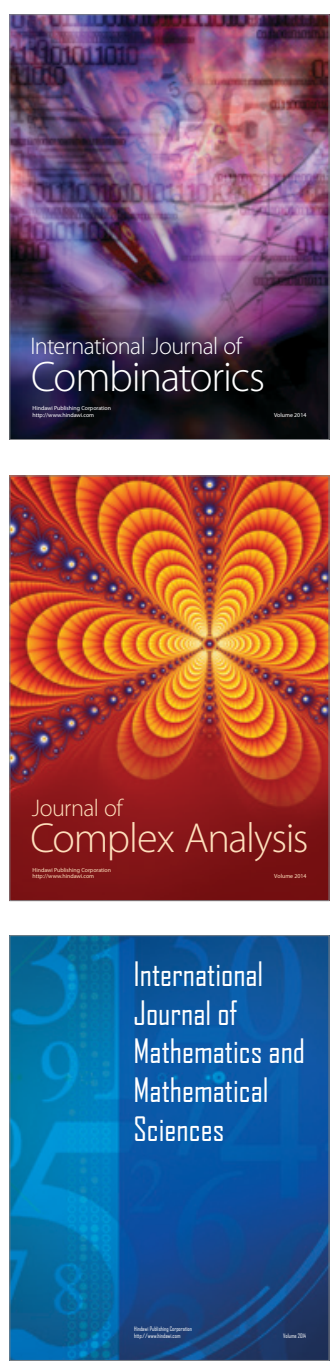
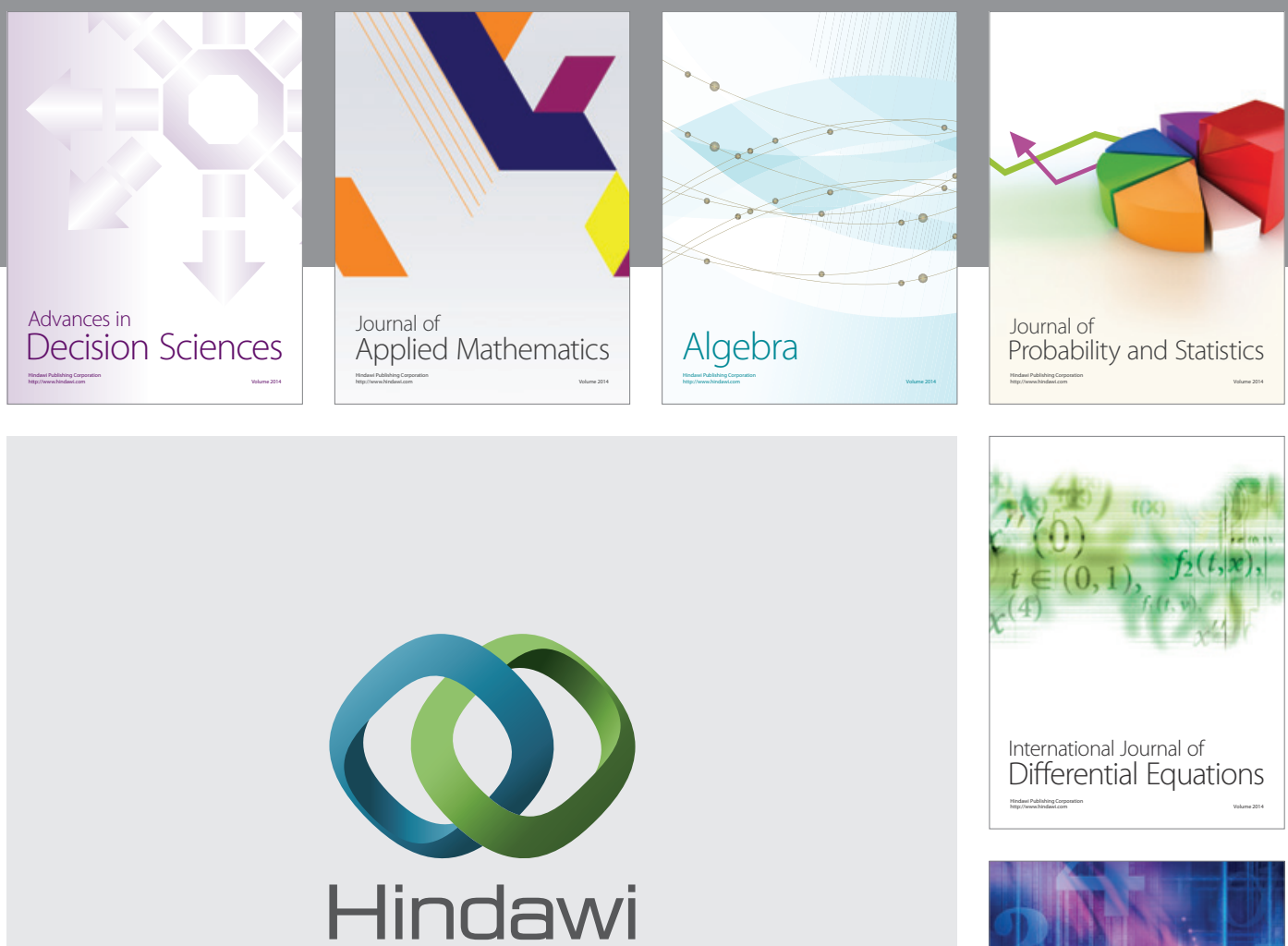

Submit your manuscripts at http://www.hindawi.com
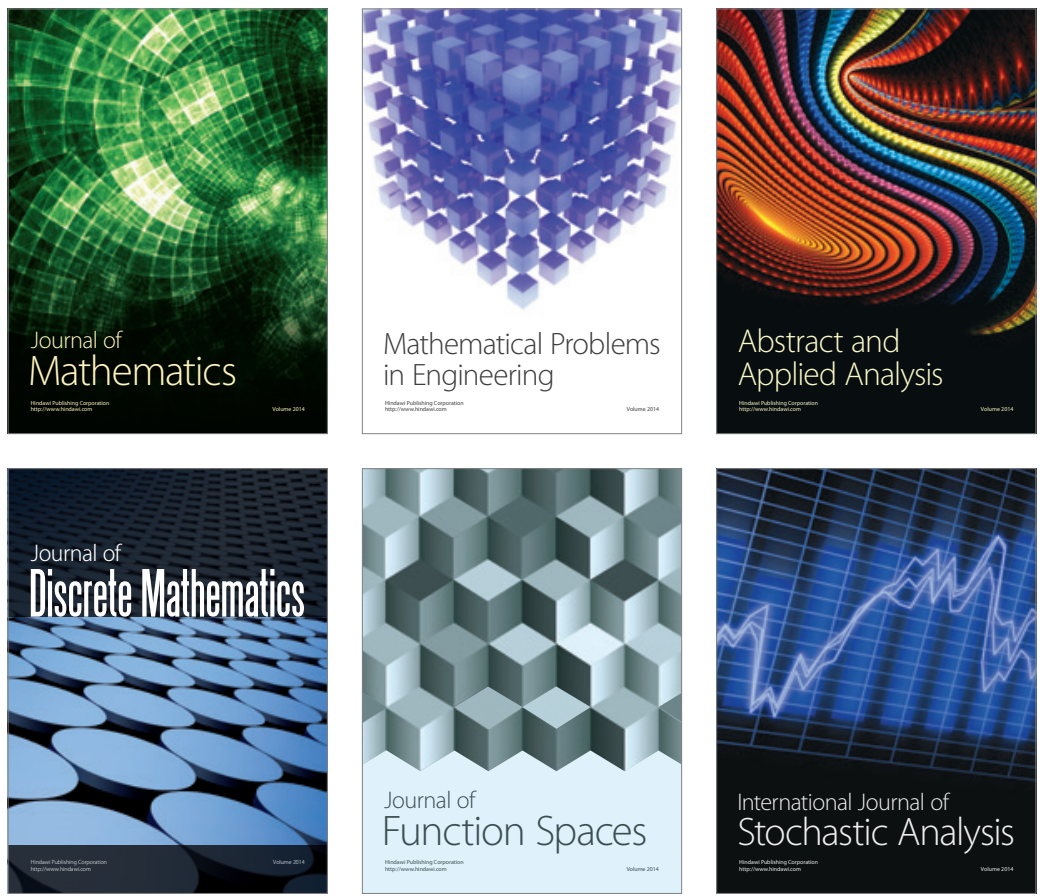

Journal of

Function Spaces

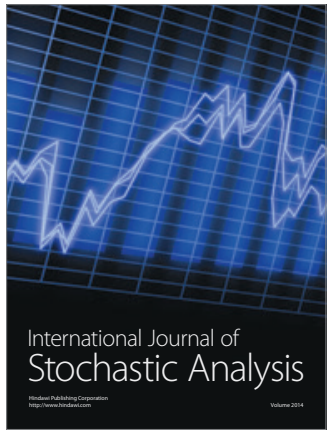

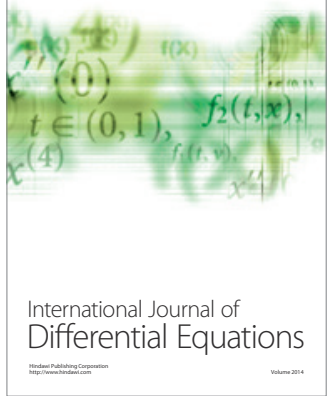
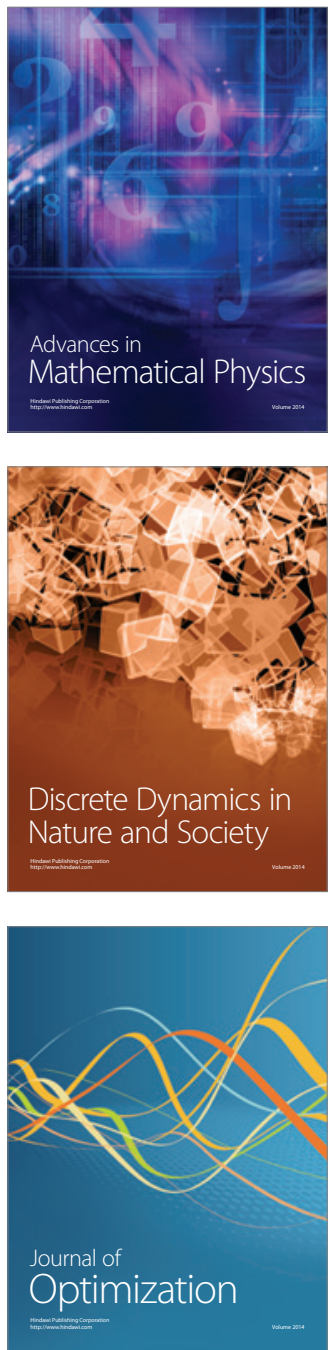